\title{
Le « non-verbal » dans l'organisation définie comme système de communication
}

\section{Claude Duterme}

\section{OpenEdition}

\section{Journals}

Édition électronique

URL : https://journals.openedition.org/communicationorganisation/2472

DOI : 10.4000/communicationorganisation.2472

ISSN : 1775-3546

\section{Éditeur}

Presses universitaires de Bordeaux

\section{Édition imprimée}

Date de publication : 1 novembre 2000

ISSN : 1168-5549

Référence électronique

Claude Duterme, « Le «non-verbal » dans l'organisation définie comme système de communication », Communication et organisation [En ligne], 18 | 2000, mis en ligne le 27 mars 2012, consulté le 05 août 2021. URL : http://journals.openedition.org/communicationorganisation/2472 ; DOI : https://doi.org/ 10.4000/communicationorganisation.2472

Ce document a été généré automatiquement le 5 août 2021 .

(C) Presses universitaires de Bordeaux 


\title{
Le « non-verbal » dans l'organisation définie comme système de communication
}

\author{
Claude Duterme
}

1 Mon propos sera de questionner ces catégories fonctionnant comme des évidences que sont le «non-verbal ». l'« organisation » ou la « rationalité ». En prenant comme base de réflexion le modèle de pensée de l'École de Palo-Alto sur la communication et en l'appliquant au champ des organisations, je voudrais revenir sur les hypothèses énoncées dans l'appel à communication de ce colloque. La question, de mon point de vue, ne sera donc pas tant de savoir comment et pourquoi le non-verbal se manifeste, mais plutôt de savoir en quoi l'existence de cette catégorie "non-verbal », ainsi que celle de « rationalité » permettent le développement de comportements et de discours managériaux, univoques et inquestionnables.

\section{Organisation et rationalité}

2 L'idée que les organisations soient fondées sur des processus rationnels n'est pas (ou plus) la seule conception possible. La lecture stratégique des organisations, par exemple, a placé un coin dans le bloc jusque-là monolithique de cette conception rationnelle ("scientifique », disait Taylor).

3 En ayant mis l'accent, depuis Taylor, sur la composante rationnelle ou rationalisable des processus de production, la dimension proprement humaine et sociale des organisations a été largement occultée par le discours organisationnel et managérial. Aborder l'entreprise comme un construit social réinstalle l'humain au cœur de l'existence des organisations.

4 Crozier et Friedberg ${ }^{1}$ nous rappellent que l'entreprise est composée d'hommes et de femmes, que leurs relations sont continues en son sein et que ces relations font l'objet d'accords partiels permettant de stabiliser provisoirement les « jeux » sociaux. Ces jeux 
se produisent dans un univers toujours limité de connaissance, ce qui restreint la rationalité des acteurs et donc du processus décisionnel dans son ensemble.

$5 \mathrm{Au}$ sein de ces jeux relativement et temporairement stabilisés ("système d'action concret») sont utilisés non seulement les personnes et leurs ressources professionnelles et sociales, mais aussi bien les dispositifs technologiques, financiers, réglementaires,... de l'entreprise.

6 En somme, si l'entreprise est dotée de systèmes techniques et de dispositifs « rationnels » tels que les décrivent et les utilisent les analystes tayloriens c'est, selon Crozier et Friedberg. parce que cela convient aux acteurs dans leur recherche de stabilisation relative de leurs jeux. Le système «rationnel » de production n'est pas constitutif de l'organisation, il en est une résultante. Il est également loin d'être aussi global que ce qu'on veut bien en dire.

\section{L'organisation comme système de communication}

7 En restant dans la ligne d'une conception de l'organisation comme construit social contingent, je voudrais proposer une approche communicationnelle de l'organisation : elle permettra aussi d'aborder la question du non-verbal dans le contexte attendu (« non-verbal, communication, organisation »).

Bateson, puis les représentants du courant qu'on a appelé « École de Palo-Alto » ont été les initiateurs de cette approche théorique sur la communication. ${ }^{2}$ En bref, les tenants de ce point de vue considèrent la communication comme un système d'interactions, dans lequel interviennent tous les signes interprétables par les acteurs dans une situation sociale.

Ce «système d'interactions » dépasse largement le cadre linguistique et paralinguistique dans lequel on avait l'habitude de maintenir les théories de la communication. Il prend en compte la totalité des comportements comme autant d'unités significatives, regroupées dans un ensemble possédant toutes les propriétés des systèmes.

10 Toute situation sociale (mettant en scène plus d'un individu) sera dès lors interprétée dans les termes d'un système de communication dont on peut dire notamment qu'il y est impossible de ne pas communiquer. En effet, tout comportement possède une valeur pragmatique significative pour qui l'observe. ${ }^{3}$

11 Par ailleurs, ce système de communication est ouvert: il est en relation avec son environnement. Pour comprendre ce système ouvert mais aussi pour pouvoir décider d'une signification des interactions à l'intérieur de celui-ci, il faudra tenir compte du ou des contextes dans le(s) quel(s) il se situe.

12 Autrement dit. la signification des interactions produites dans un système de communication dépend aussi du ou des contextes pertinents pour ce système et, en ce qui concerne les acteurs, de leur (re)connaissance des indicateurs de contexte.

13 Enfin, si nous réfléchissons en termes de système, la question du feed-back devient centrale dans la description des phénomènes. À toute unité significative de communication (à tout comportement) font nécessairement suite une rétroinformation, puis une rétroaction, de sorte qu'il nous faut considérer le système 
suivant une logique circulaire et non plus linéaire si nous voulons lui attribuer une signification.

$14 \mathrm{Au}$ sein des systèmes relativement stables dans le temps et par exemple au sein des organisations, les individus concernés vont sélectionner à travers leurs interactions un certain nombre de comportements « favorables » ou « intéressants » pour eux et, par là même, en négliger d'autres. Pour un observateur extérieur, ces sélections se manifestent par l'apparition de redondances comportementales.

15 À un niveau logique supérieur, on pourra observer des "patterns " ou modèles de redondances. Ceux-ci agissent comme des mesures de stabilisation du système autour de certaines normes, ce qui assure son équilibre homéostatique. Ces figures de régulation ne sont généralement pas perceptibles par les individus - éléments du système. Elles fonctionnent comme des règles du jeu mises en place petit à petit à travers la succession des interactions, mais non portées à la connaissance explicite des membres du système. Les individus n'en deviennent d'ailleurs relativement conscients que lorsqu'ils changent de contexte et s'aperçoivent que les règles dans le nouveau contexte ne sont pas les mêmes que dans l'ancien. Il s'agit donc de " ce qui se fait et ne se fait pas » au sein du système considéré et qui se fait connaître non particulièrement sous la forme verbalisée des règlements, par exemple, mais essentiellement à travers les comportements des uns et des autres et intériorisé dans ces comportements. Cette non-verbalisation est d'ailleurs un élément constitutif d'une certaine souplesse dans le suivi quotidien de ces contraintes.

\section{Quelle rationalité ?}

Rappelons que ces figures de redondances sont la condition du maintien du système en équilibre homéostatique. Il s'agit donc bien de réponses rationnelles à un état: l'établissement de régularités viables et acceptables par tous au sein d'un ensemble qui serait autrement totalement chaotique. Les redondances font sens et équilibre au sein des organisations. Les comportements des individus, une fois qu'ils sont analysés dans leur contexte pertinent, sont donc toujours rationnels. 11 se peut cependant que, en regard d'un autre contexte (plus large ou plus étroit), ces comportements fassent obstacle à d'autres régularités ou changements. Cela n'en fait pas des comportements irrationnels en soi pour la cause.

Parler de rationalité dans ce cadre n'apporte donc pas d'information particulière, ni en soi ni en comparaison à d'autres systèmes de communication. Lorsqu'on parle de la rationalité de l'organisation, on parle alors d'une forme particulière de rationalité, celle que Taylor a qualifiée de scientifique et qu'on ne qualifie plus maintenant tant elle passe pour être "normale ». Somme toute, une forme de rationalité qui tend à limiter les possibilités d'interventions non programmées des membres de l'organisation et à y substituer une planification plus globale. C'est celle-là qui s'impose comme un « donné » et tente de revêtir le masque du naturel, du non construit.

C'est donc dans ce cadre de programmation des comportements que la rationalité est liée à cette catégorie particulière de comportements qu'on appelle le «langage ». Celuici serait en quelque sorte le support par excellence de l'organisation de la rationalité dans l'entreprise. 


\section{À la recherche du non-verbal}

19 La catégorie de phénomènes regroupés sous le vocable «non-verbal » apparaît comme un repérage " en creux » : comme l'expression le fait d'ailleurs savoir, on désigne par là les éléments identifiés comme annexes dans les actes de communication puisque le fondement de la communication serait le verbal. Cette vision de la communication relève d'un point de vue où seule la transmission d'informations est considérée comme pertinente. Dans ce cadre, le centre de l'attention est en effet focalisé sur le verbal et ses diverses déclinaisons. Le non-verbal apparaît comme une sorte de comportement parasite lié à la transmission d'informations et dont on se passerait bien, mais qu'il faut néanmoins prendre en compte puisqu'il ne peut manquer de se manifester.

Par contre, si on s'attache aux phénomènes de communication dans une perspective systémique et pragmatique, le point de vue change de manière substantielle.

21 On l'a dit, dans les systèmes de communication tous les comportements sont porteurs de signification parce qu'on ne peut manquer de les interpréter (« on ne peut pas ne pas communiquer ») à l'intérieur du contexte où ils se produisent. Le verbal constitue une des manières possibles de se comporter - une manière particulièrement évoluée et complexe, sans doute, mais certainement pas unique ni même centrale dans le champ des interactions, et donc de la communication.

Par ailleurs, à travers l'ensemble des comportements possibles au sein des contextes disponibles, on peut décrire deux modes de communication : le mode digital et le mode analogique.

Le mode digital correspond à toute forme particulière de comportement qui vise à transmettre des significations (des éléments d'information) les moins ambiguës possibles. Dans cette forme de codage de l'information, le signe est très éloigné du signifié, l'abstraction minimisant les risques de confusion sur le sens.

Le mode analogique, par contre, est une forme de communication plus proche de l'image, de la comparaison, de la reproduction et de la copie du signifié. La richesse de ce mode de communication est très grande mais les risques de confusion et d'indécision sur le sens en sont fortement augmentés.

Remarquons que le mode digital peut être le fait d'éléments de communication autres que le langage. La chaîne de montage, telle qu'elle a pu naître des recherches de Taylor, ne laisse pas de place à l'interprétation: un ouvrier par machine, une machine par geste/opération, la chaîne détermine l'ordre des opérations ainsi d'ailleurs que le temps imparti par opération. Les risques d'ambiguïté sont drastiquement réduits.

On le voit, le non-verbal ne relève pas nécessairement du mode analogique de la communication. Je pense qu'au contraire, les efforts déployés actuellement dans une série de domaines du management visent à faire porter un grand nombre de messages digitaux à travers des supports non-verbaux. Bien sûr. il s'agit là d'un "non-verbal » particulier puisqu'il est inscrit dans des comportements en quelque sorte figés : des machines, des programmes, des éléments architecturaux...

Les catégories «verbal » ou «non-verbal» ne sont dès lors pas centrales lorsqu'on décrit l'organisation en termes de système de communication. En fait, elles ne sont déterminantes que dans un cadre où la communication est envisagée du seul point de vue de la capacité à transmettre des informations (contenu) et où les relations (interactions) entre les personnes ou les groupes ne sont pas prises en compte. 
28 Dans l'organisation-système de communication, la plupart des comportements et indicateurs de contexte sont non-verbaux: l'agencement des locaux, le type de fournitures qui y circulent, les systèmes techniques qui y sont à l'œuvre, les routines de travail, etc. Or, il faut bien remarquer que ce sont pour l'essentiel ces comportements et indicateurs de contexte qui définissent pour les acteurs la manière dont ils doivent se comporter dans l'entreprise. ${ }^{4}$ Ce sont eux qui décrivent la tonne de rationalité à l'œuvre dans cette organisation particulière. Le "verbal» est une composante finalement assez marginale de cette masse de comportements qui traduisent la rationalité: des ordres directs lorsqu'ils sont nécessaires, des rappels du cadre (affiches, règlements, etc.) et autres notes de services.

29 Par contre, l'ensemble de ces indicateurs de contexte et de ces comportements standards et routiniers sont fortement digitalisés. C'est parce qu'ils laissent peu de place à l'interprétation qu'ils agissent comme des cléments « rationalisants » ou plus exactement donneurs d'ordre.

\section{Indice et ordre dans le système de communication}

À partir du moment où la communication est définie comme un ensemble de comportements en interaction, le seul fait de transmettre ou non une information, de le faire de telle ou telle manière en privilégiant tel ou tel média... constitue un fait informationnel de première importance. Autrement dit. l'acte et la manière d'informer - ou non - déterminent la façon de comprendre l'information transmise ou l'absence de cette transmission.

Nous touchons là aux aspects «indice» et "ordre» impliqués dans toute communication. Tout comportement implique à la fois une dimension d'ordre, d'indication sur la manière de comprendre le message, et le message lui-même, son contenu. L'aspect d'ordre est nécessairement lié à la nature de l'interaction en cours : il porte toujours sur une définition, le plus souvent implicite, du type de relation qui se noue entre les acteurs de la communication. Au bout du compte, cela revient à signifier : "voilà comment nous devons comprendre la nature des messages échangés entre nous ». L'aspect « indice» ou contenu de la communication ne prend à son tour de valeur qu'en fonction du type de relation impliqué. Ajoutons qu'une grande partie des comportements effectués le sont en vue de confirmer et de valider le type de relations en cours, généralement implicitement d'ailleurs.

Par exemple, un chef de service pourra donner un ordre à l'un de ses employés sans que cet ordre soit dicté par des impératifs "rationnels" liés à la production. Mais ce faisant, il rappelle et confirme l'état des relations en cours.

Si on revient maintenant au concept de rationalité et à son origine taylorienne, on peut analyser son apparition comme un fait communicationnel. Le premier message, le plus fondamental, est de l'ordre de la définition de la relation: l'acte même d'instituer l'organisation scientifique du travail revient à donner unilatéralement à l'analyste, donc au patron, la capacité à ordonner la manière dont le travail va s'effectuer, et par là aussi à en enlever la plus grande part à l'ouvrier. Il indique également l'état des relations en cours : relation complémentaire forte et rigidifiée par cette action même. 


\section{Positions complémentaires et digitalisation de la relation}

34 Il apparaît que le type de système de communication "entreprise » centré sur une régulation de type complémentaire tend à se renforcer et à se rigidifier actuellement grâce à la digitalisation de la "relation » (l'aspect " ordre » de l'interaction). Ceci se marque aussi par l'intégration des consignes, des contraintes et du contrôle de ces contraintes dans le processus de production lui-même, y compris à travers le rôle dévolu aux opérateurs. Ils doivent en effet de plus en plus veiller eux-mêmes à la bonne application du contrôle de leurs opérations. Le contrôle d'ensemble étant attribué au système technique : ordinateurs, chaîne de production, progiciels de gestion (SAP par exemple),...

Un effet particulièrement significatif de ce processus du point de vue communicationnel est d'éloigner un des interlocuteurs de la relation - celui qui, justement, a pris l'initiative de l'imposition rigide de la complémentarité. Il y a de moins en moins de chefs auprès desquels il serait possible de chercher à contester le mode d'interaction à l'œuvre; ceux qui restent se sentent ou se disent aussi démunis que les opérateurs de base. Non seulement la relation complémentaire est-elle rigidifiée à travers cette opération mais la capacité des autres partenaires de la relation à la contester est, de son côté, amoindrie par la distanciation et la difficulté d'identification du partenaire one - up.

C'est, en dépit de tous les discours (le verbal) ambiants, dans cette direction qu'on peut voir se diriger une grande partie des efforts actuels dans la gestion des entreprises et des flux de production, jusque et y compris la gestion des « ressources humaines ». Si la ligne hiérarchique peut être raccourcie et les organigrammes aplatis, c'est bien parce que la transmission des ordres ne se fait plus de manière verbale mais à travers l'information incluse dans les processus. Le développement de la standardisation et de la certification (normes Iso, etc.), en cours depuis un certain temps maintenant, est conforme à la logique taylorienne dont on ne cesse par ailleurs de répéter (message verbal) l'éternelle disparition. Les techniques modernes et notamment les capacités de transmission et de stockage de l'information ont en effet permis de se passer de l'intermédiaire de la hiérarchie pour imposer et vérifier le circuit du travail et l'adéquation de la production aux normes imposées. La disparition des petits chefs et l'intégration de plus en plus poussée de l'aspect "ordre " au sein des dispositifs de production eux-mêmes constitue une avancée logique et particulièrement performante dans la perspective taylorienne rationnelle. Plus les ordres sont intégrés au processus de travail lui-même, moins on laisse d'ambiguïté dans l'interprétation de ces ordres. C'est cette même logique qu'on retrouve dans la normalisation liée aux démarches qualité : après avoir touché les processus de production dans leur conception et leur mise en place, la normalisation intervient sur la formation des opérateurs et vise à s'assurer de la standardisation de leur comportement, y compris dans l'auto-contrôle de leurs propres actions.

On voit donc s'installer avec de plus en plus de force la tendance à la digitalisation du « non-verbal », par toutes sortes de moyens qui relèvent de la capacité à obtenir, traiter et transmettre des informations. Les ordinateurs (par exemple) ajoutent à leur statut d'outil et de poste de travail celui de contrôleur (enregistreur) automatique du comportement des opérateurs. 

fait. Ils appliquent, à travers les opérations qu'ils réalisent, le type de relation que les responsables de leur programmation souhaitent imposer au sein du système de communication. On voit combien la relation est distanciée : par rapport au partenaire absent, le dispositif technique apparaissant comme déjà là.

\section{Communication interne et déni de la position complémentaire}

Au sein des organisations, la tendance est donc à l'instauration unilatérale d'un type de relations complémentaire rigide et à la mise en place de processus tendant à éviter la possibilité de remise en cause de cette définition de la relation. C'est cette logique relationnelle qui est au cœur du credo de la rationalité des entreprises.

Or les systèmes de communication, pour assurer leur survie et leur équilibre à terme, doivent opérer sur des bases de régulation garantissant un minimum de souplesse en leur sein. Autrement dit, il devrait être nécessaire d'assurer à chacun des partenaires de la relation la possibilité, même réduite, de sortir parfois de sa position basse pour soit passer en position haute, soit établir (même ponctuellement) une relation de type symétrique. Ces modifications dans la nature des interactions sont théoriquement possible entre des personnes, capables de modifier volontairement leurs relations. Mais cela ne l'est plus quand la régulation de ces interactions a été internalisée dans des processus automatiques.

Dès lors, il est logique de voir fleurir et s'épanouir les appels à la communication interne comme vecteur de mobilisation. Il s'agit «d'expliquer le sens» des changements, de « donner la parole » aux « collaborateurs »... En bref, de rétablir dans le discours (langage verbal) des pans d'égalité et de relation symétrique qui ne peuvent s'insérer dans les comportements quotidiens, bloqués par des processus qui maintiennent rigidement la position complémentaire.

comunication interne conçue comme la production de messages (et non, encore une fois, comme l'ensemble des interactions inhérentes au système de communication ou de ses figures de régulations) doit alors servir au management pour tenter d'équilibrer la relation ou de le faire croire. Le partenaire en position one-up cherche à métacommuniquer pour qualifier la relation comme non rigidement complémentaire.

Ce faisant, il accentue en fait cette rigidité : c'est encore lui qui tente d'imposer un sens aux comportements qu'il rend par ailleurs univoques. De ce point de vue, l'expression pensée unique paraît tout à fait adaptée comme description de la situation communicationnelle.

la mesure où cette tendance s'accentuerait et se généraliserait, il resterait aux membres de l'organisation deux possibilités s'ils veulent tout de même équilibrer la relation.

Soit ils réagissent dans le champ des comportements organisationnels eux-mêmes. Mais ceux-ci deviennent de plus en plus inaccessibles aux opérateurs puisqu'ils sont intégrés aux processus et aux dispositifs techniques. Comme la marge de manœuvre est quasi inexistante, reste alors la possibilité de tenter de supprimer le support de digitalisation des comportements. C'est la voie ouverte au sabotage, comportement non-verbal s'il en est. Le sabotage réfute à la fois le mode de relation rigidement

Communication et organisation, 18 | 2000 
complémentaire imposé par le dispositif technique et le discours sur le sens, la logique de travail unilatéralement définie. Ces aspects non-verbaux (comportementaux) des autres partenaires, porteurs de contestation, seront logiquement qualifiés de non rationnels par le partenaire one-up.

Soit ils cherchent à investir dans le champ du discours une position qui ne leur est pas accessible dans le champ du comportement au travail, en espérant y gagner un positionnement différent et plus équilibré. Mais s'ils obtiennent le droit à la parole dans le champ des entreprises, cela supposerait à terme la possibilité de contester la nature de la relation; l'autre partenaire est-il prêt à lâcher la position one up ? Il me semble qu'on aborde là une question fondamentale dans la réflexion sur la « communication interne » des organisations.

\section{NOTES}

1. Crozier M. et Friedberg E, L acteur et le système. Seuil, 1977 et Friedberg E., Le pouvoir et la règle. Seuil, 1993.

2. Cfr. Notamment : Winkin Y., La nouvelle communication, Paris, Seuil, Points, 1981.

3. Cfr. Watzlawick et al., Une logique de la communication, Paris, Seuil, Points, 1972.

4. Cfr. de Saint-Georges, Pierre. «Stratégies culturelles d'entreprise: une approche par la communication ». Culture d'entreprise, vous avez dit cultures dossier $\mathrm{n}^{\circ} 12$ de l'Institut des Sciences du travail pp. 89-99, juin 1990, UCL, Louvain-la-Neuve,

\section{AUTEUR}

\section{CLAUDE DUTERME}

Centre d'Études de la Communication, Université Catholique de Louvain 